\title{
The Role of the Speech-Language Pathologist in the Schools for the Treatment of Voice Disorders: Working within the Framework of the Individuals with Disabilities Education Improvement Act
}

\author{
Bari Hoffman Ruddy, Ph.D., ${ }^{1,2}$ Vicki Lewis, M.A., ${ }^{2}$ and \\ Christine M. Sapienza, Ph.D.3
}

${ }^{1}$ Department of Communication Sciences and Disorders, University of Central Florida, Orlando, Florida; ${ }^{2}$ The Ear Nose Throat and Plastic Surgery Associates Voice Care Center, Winter Park, Florida; ${ }^{3}$ Department of Speech, Language, and Hearing Sciences, Brain Rehabilitation Research Center, University of Florida, Malcom Randall VA, Gainesville, Florida.

Address for correspondence: Bari Hoffman Ruddy, Ph.D., P.O. Box 162215, Orlando, FL 32816

(e-mail: bhruddy@mail.ucf.edu).
The role of the speech-language pathologist (SLP) has developed considerably over the past 20 years given the medical and technological advances in life-sustaining procedures. Children born with congenital, surgical, or "medically fragile" conditions become mainstreamed into regular school-based settings, thus extending the traditional role of the treating SLP and multidisciplinary team. Understanding the impact of associated voice disorders on educational performance requires dissemination of additional and important information, as eligibility decisions for students in school-based settings must be made within the framework of the federal legislation and regulations governing the provision of services for students with disabilities. This article discusses how to identify children with voice disorders under the Individuals with Disabilities Education Improvement Act (IDEA), the role of the SLP in various triaging scenarios, and how models of voice therapy can be integrated in a school-based setting.

KEYWORDS: Child, student, voice disorder, IDEA, treatment, education

Pediatric Voice; Guest Editor, Katherine Verdolini Abbott, Ph.D., CCC-SLP

Semin Speech Lang 2013;34:55-62. Copyright (C) 2013 by Thieme Medical Publishers, Inc., 333 Seventh Avenue, New York, NY 10001, USA. Tel: +1(212) 584-4662. DOI: http://dx.doi.org/10.1055/s-0033-1342976. ISSN 0734-0478. 
Learning Outcomes: As a result of this activity, the reader will be able to (1) explain how eligibility decisions are made within the framework of the Individuals with Disabilities Education Improvement Act (IDEA), (2) describe the role of the speech-language pathologist (SLP) in the evaluation and management of children with voice disorders under the definition of IDEA, (3) list the ways that voice disorders can impact educational and extracurricular performance in children and adolescents.

The role of the speech-language pathologist (SLP) in treating childhood voice disorders in the schools has developed considerably over the past 20 years. The clinical assessment of voice disorders in the schools requires identifying the role of certain disease processes and influence on their management. Technological advances have sustained the life of the medically fragile child and have diagnosed congenital conditions in utero. Over time, the medically fragile child becomes mainstreamed into regular school-based settings, thus extending the traditional role of the treating SLP and multidisciplinary team.

Given these advances, many schoolbased SLPs are expected to assess and treat larger numbers of children with voice and other upper airway disorders than previously. Many SLPs have expressed trepidation in treating this population because their graduate-level education in this area is minimal and their clinical exposure is limited or nonexistent. Their lack of confidence in treating this population may lead to nonidentification and nontreatment of these disorders. In particular, the true impact of dysphonia on the lives of children is currently unknown and warrants study. ${ }^{1}$

Many SLPs have also reported uncertainty as to what types of voice disorders are educationally relevant to treat in the school-based setting. Interview and survey outcomes indicate that "eligibility decisions under the definition of the Individuals with Disabilities Education Improvement Act (IDEA) are left to interpretation, causing further confusion and inconsistency in practice for the school-based clinician." ${ }^{2}$ For example, some of the questions that have been raised from the interviews include: What types of voice disorders are educationally relevant to treat? Are all voice disorders that occur in the school-aged child educationally relevant to treat? Can voice difficulty in the school-based setting actually limit academic goals and achievement? To answer these questions and understand the role of the SLP, knowledge about these legislative acts is very important.

\section{DEFINING IDEA}

Eligibility decisions for students in schoolbased settings must be made within the framework of the federal legislation and regulations governing the provision of services for students with disabilities. The Individuals with Disabilities Education Improvement Act of 2004 (IDEA 2004-P.L. 108-446) was formulated to work together with another federal education law, the Elementary and Secondary Education Act (ESEA; more recently referred to as the No Child Left Behind Act of 2001). ${ }^{3}$ ESEA provides educational programming regulations for all students and IDEA targets individual students with needs including special education or related specialized services. One of the four rights afforded by IDEA is "related services," which includes SLP intervention. IDEA requires that students with special needs have an individualized education plan that outlines treatment goals and is developed by a multidisciplinary team of education professionals, including the SLP and the student's parents. Under the law, voice disorders constitute an educational disability. However, students with voice disorders may fail to receive services because it is thought that their disability does not adversely affect academic performance. ${ }^{1,4}$ In a letter of interpretation, the Office of Special Education Programs clarified the term educational performance, as used in IDEA, "to include effects upon academic and nonacademic areas. Furthermore, if the presence of a speech-language impairment has been established by a SLP through appropriate appraisal procedures, the receipt of services is not conditional upon academic performance. A child who is achieving at grade 
level can still qualify as having a speechlanguage disability."4(p.593)

\section{IMPACT OF VOICE DISORDERS ON EDUCATION}

A voice disorder can include distinct vocal behavior related to the pitch, loudness, and/or overall quality of voice and can vary by frequency and consistency. Some of the common voice disorders in school-age children may occur as a result of so-called functional, structural, or neurologic processes. Some of the common functional disorders that occur from phonotraumatic behaviors (i.e., vocal behaviors causing tissue damage) include vocal fold edema, vascularity, nodules, and/or polyps. Fluency or articulation disorders may also result in secondary functional voice problems due to increased phonatory effort. ${ }^{4}$

Other structural voice disorders can be congenital or acquired and may include papilloma, medical conditions such as asthma, laryngopharyngeal reflux resulting in cough (a disorder in itself) and also resulting in laryngeal trauma as a result of the cough, as well as granuloma or contact ulcers. Some neuromuscular disorders with a secondary voice component include cerebral palsy, muscular dystrophy, or other disease processes affecting motor control. Structural changes to the upper airway (congenital or acquired) can also impair laryngeal function. Congenital conditions include laryngeal anomalies, such as stenosis, laryngomalacia, clefts, laryngoceles, webs, and cysts.

Central nervous system disorders include hydrocephalus, encephalocele, and ArnoldChiari syndrome; disorders of the cardiovascular system may include cardiomegaly or abnormal vessels. Acquired disorders include paralysis or paresis; paradoxical vocal fold motion; trauma from birth injury or postsurgical correction of cardiovascular, esophageal, or cranial anomalies; tumor compression; and infection, such as whooping cough, polio, syphilis, or tetanus. ${ }^{5,6}$ The challenge for clinicians is to understand how the underlying pathophysiology of these disease processes affects vocal function and the student's behavior to provide effective therapeutic interventions.
School-aged children with these types of voice disorders can be negatively affected in a variety of ways with regard to reaching or meeting their educational goals. For example, students may attempt to conceal atypical vocal production, or they may have feelings of inferiority about their voices. This in turn may seriously limit their classroom participation, giving them fewer opportunities to practice and receive teacher feedback. Andrews stated that school-aged children's preoccupation with concealing deviant vocal behaviors might interfere with concentration during academic activities and/or cause peer reaction or embarrassment. ${ }^{4}$ Social-emotional implications may include students becoming withdrawn and reticent or vocally aggressive and defiant in situations in which the student is attempting to compensate for a vocal disability. Children and teens reported feeling that their dysphonia received "undue attention" and reduced their participation in events because of voice. Additionally, findings for toddlers (parent interviews) indicated functional/physical issues, such as "running out of air" and "sometimes voice does not work." These problems can become progressively worse without intervention and can seriously impact learning. ${ }^{1}$

Additionally, children who use a limited number of vocal strategies (such as whining, talking loudly, and incessantly) as a way to solve interpersonal problems may be at risk for being evaluated in negative ways by educators. This may indirectly affect how they are viewed in all aspects of their educational process.

For the adolescent populations, academic content emphasizes school-to-career activities. The school-to-career program connects academic learning to practical application. Many of these career-related activities demand efficient vocal communication skills for interviewing, employment opportunities, internships, apprenticeships, or mentorships to create a strong relationship between the student and the worksite. The adolescent with a voice disorder may have fewer opportunities (or none at all) to participate in these educational routines. Furthermore, adolescents with voice problems may have difficulty modifying maladaptive habits and inappropriate compensations later in life as they transition into collegeand career-related activities. ${ }^{4}$ 
Some of the adverse effects of voice impairment for these age groups can include:

- Difficulty being heard or communicating in educational environments inside or outside of the classroom setting

- Limited participation in public speaking activities

- Fear of participating in oral reading activities

- Limited participation in classroom discussion with peer groups

- Fear of conversing in interpersonal interaction (i.e., raising hand to request to go to the bathroom)

- Limited participation in regular physical education routines due to compromised physiological aspects of the laryngeal anatomy

- Limited participation in music education (vocal and instrumental) due to upper airway compromise

- Reluctance to participate in activities such as school plays, cheerleading, debate, and so on

- Limited participation in secondary education co-op activities requiring the student to take nonvocal jobs only

- Reluctance to participate in interview activities, thus limiting their access to employment and certain educational opportunities

- Negative attention from peers, teachers, and other school personnel

- Hindrance of academic goals of other classroom students (A disordered voice quality may be distracting to other classmates who focus on the abnormal voice quality instead of the content of the message.)

\section{IDEA: THE MULTIDISCIPLINARY TEAM}

According to IDEA, a multidisciplinary team must decide eligibility for special education services. The assessment must be completed in the student's "native" language. Members of the team include:

- The student's parents

- At least one regular education teacher (if the student will be participating in regular education)
- At least one special education teacher or special education provider

- A representative of the school

- An individual who can interpret the instructional implication of evaluation results

- The student

- Ad hoc: the otolaryngologist and voice pathologist and/or vocologist for the student who may be involved in professional voice development

Each member serves a critical role on the multidisciplinary team. For example, the role of the classroom teacher is to identify students at risk or to have an understanding of the consequences of vocal difficulty on a student's educational performance. In addition, the classroom teacher works in collaboration with the SLP by providing consistency between the therapy room and the classroom for generalization into the student's more natural setting (i. e., in the classroom with peers, on the playground, and in the lunchroom). Classroom teachers are able to facilitate and support the student's efforts to maintain good vocal habits throughout the school day versus the portion of time that the student is receiving direct services.

Because voice therapy relies on home programming for carryover to the student's real world, the compliance and motivation of $\mathrm{pa}^{-}$ rents, siblings, friends, and other family members are critical to treatment success. Occasionally the student may face teasing or ridicule because of the need to practice various vocal exercises or restrict the use of voice. Therefore, the inclusion of family members and friends in the student's vocal rehabilitation program is a key factor in generating support of the therapy process when the student leaves the therapy setting. Without this support, the student may feel alone or misunderstood in the effort. SLPs can help by counseling the parent, sending home checklists, and following thorough with phone or face-to-face conferences to facilitate home carryover. Another role of the parent is to assist the SLP in identifying phonotraumatic behaviors occurring in the home or extracurricular settings, which may include loud peer or family interactions, smoky environment, vocal manipulations (i.e., young 
child crying to get what he or she wants), and possible allergens.

In short, the role of the SLP in the schoolbased setting is vast. The SLP has become an advocate for the student and may be motivator for the entire team. The SLP is typically the only on-campus link between the physician and parent, and as such the SLP acts as the leader for dissemination of information among all team members. Most importantly, the role of the SLP is to be an educator for campus staff and teachers. The SLP may do so by providing inservices, demonstrations, checklists, and/or pamphlets. In turn, teachers and staff can help identify students at risk and make appropriate referrals in a timely manner.

In many settings, once the school-based personnel identifies a student as having a "potential voice problem," it is the school SLP who often becomes the primary advocate for the accomplishment of the student's laryngeal examination. The justification for persistence in this recommendation is clear-cut. For example, there are times when dysphonia in a child may seem consistent with a hyperfunctional disorder, but in fact it may be a perceptual representation of other organic pathology (e.g., papillomatosis, submucosal cysts, gastrointestinal or laryngeal-pharyngeal reflux, webbing, stenosis, paralysis, etc.). Medical rather than solely behavioral treatment is required for these laryngeal conditions (e.g., see Glaze ${ }^{7}$ ). Moreover, an understanding of the primary pathology will lead to better insight regarding the secondary laryngeal and respiratory compensations that a student may exhibit.

There is also a need for the SLP to "suspect a correct diagnosis" (e.g., see Glaze ${ }^{7}$ ) whenever the student's vocal behaviors and verbal affect do not match the profile of "vocal abuse/misuse" (terms now preferably substituted with more descriptive and less emotionally loaded ones) or other patterns associated with a particular disease process. ${ }^{8}$ For example, Glaze discussed an unusual case that was described by a clinician regarding a child with presumed nodules, when the child actually had suffered a fractured larynx during an incident of stranger abuse. Because the child was very reticent, very quiet, and withdrawn, the clinician was alerted to the mismatch between typical hyperfunctional voice activity and her client's vocal behaviors. ${ }^{7}$

Another example, described by a referring otolaryngologist, found that a child had been treated in the school-based setting for "presumed vocal nodules" due to the perceived "hoarse voice quality, strained production, and inadequate respiratory dynamics." The child had been receiving "speech therapy" in the elementary school for three and a half years before a diagnosis of anterior webbing was made. These scenarios provide a firm rationale for insisting that all children with presumed voice disorders must receive an otolaryngologist's medical diagnosis prior to initiating any therapeutic services. ${ }^{9}$

\section{TRIAGING SCENARIOS}

\section{Scenario 1}

When a student is referred to the school-based SLP, the typical scenario (scenario 1) involves an abbreviated screening of the student's vocal quality. If the screening reveals moderate to severe changes in voice quality, the SLP will then meet (school district/state) criteria to refer the student to a physician for a laryngeal examination (preferably a laryngologist). Following the laryngologist's exam, the student is referred back to the school-based SLP with a diagnosis of laryngeal pathology and a recommendation for voice therapy services. However, there are a few triaging scenarios that are more complex, which further extend the role of the SLP. Some of these scenarios may include the following.

\section{Scenario 2}

- A student is referred to the SLP by classroom teacher.

- The SLP administers screening.

- The student does not qualify for further evaluation (based on district/county guidelines).

How far does the role of the SLP extend? Could the student's "mild" voice difficulty worsen 
without treatment? Should the SLP refer the student for private services?

The SLP should include a follow-up with a parent to provide a checklist of behavioral indicators in case the condition worsens. Certainly, the student's condition could get worse over time particularly if no behavioral management is completed and the parent is unaware of how to modify the home environment. The SLP may elect to educate the parent on vocal health and hygiene and organize a home therapy program. The SLP should also provide the parent with resources available in the community if the parent wishes to seek services outside the school setting.

\section{Scenario 3}

- A student is referred to the SLP by classroom teacher.

- The SLP administers screening.

- The child is referred to physician for laryngeal evaluation.

- The student is never examined by a physician due to noncompliance by parent or no financial support.

- How do the student's needs get addressed under IDEA?

In several counties or school districts, scenario 3 has been successfully addressed by the schoolbased SLPs organizing free community-based clinics. These clinics involve a physician (preferably laryngologist) who volunteers his or her time for a brief examination and performs an indirect (or preferably endoscopic) laryngeal examination for the children who were identified through school screenings. These clinics have proven successful when accomplished through collaborative efforts of a laryngologist and SLP specializing in voice care and particularly knowledgeable of the distinguishing features of pediatric laryngeal structure and function. In this regard, an SLP and a laryngology team can work with the school-based SLP, parent, and child to render a diagnosis and implement therapeutic services under the guidelines of IDEA.

\section{Scenario 4}

- A student is referred to the SLP by classroom teacher.

- The student's screening from SLP warrants a referral to the laryngologist.

- The laryngologist report is negative for pathology but agrees that the child has "functional" dysphonia.

- What is the role of the SLP? Will the student need therapeutic services or will he or she just outgrow the behavior?

Scenario 4 may present some challenges to the school SLP, primarily because school district/ state guidelines may vary regarding the management of strictly "functional" cases, without associated organic findings. If school district/ state guidelines do not permit the SLP to initiate therapy, one possible solution is to counsel the classroom teacher on therapeutic strategies that may be helpful in eliminating deviant or functional behavior. Another strategy is to determine if the student is stimulable for any healthy/normal voice production. If the student is stimulable then the SLP may elect to provide the parent and/or classroom teacher with strategies to facilitate the therapeutic process. Last, the school-based personnel may consider the potential for another underlying cause for the resulting voice quality and make appropriate referrals to medical professionals to share in the evaluation and care of this student. This could include the school psychologist.

\section{Scenario 5}

- A student is referred to the SLP by classroom teacher.

- The student's screening from SLP warrants a referral to the laryngologist due to presumed hyperfunction.

- The laryngologist finds organic pathology (or severe structural abnormality) and recommends voice therapy.

- The SLP feels uncertain about the kinds of voice therapy that are appropriate for this student and is uncomfortable initiating treatment. 
- How does the student receive the services that he or she requires?

It may be helpful to identify an "SLP voice specialist" within the school district who can consult on these difficult-to-treat cases. Inservices that are presented in a "grand rounds" style may also be helpful so that ideas for treatment can be discussed or demonstrations of new treatment techniques can be practiced in a forgiving environment.

The child who experiences paradoxical vocal fold motion disorder (also colloquially called, "vocal cord dysfunction") or other disorders associated with upper airway limitation may represent a significant challenge for some school-based SLPs. These conditions require special attention in education and treatment considerations. These cases can experience difficulty breathing during exertion and in stressful situations at school; intermittent audible stridor can be noted, especially during physical education classes upon exertion. One recommendation is for the school-based SLP to communicate with the physician who made the diagnosis to gain a better understanding of this specific disorder. If the student was seen by an SLP at the physician's office, it would be of significant benefit to discuss the assessment and the specific recommendations made for treatment. The school SLP should educate and work closely with school-based personnel who interact with this student including the physical education teacher(s), classroom teacher(s), and the school nurse. This may provide school personnel/educators with a better understanding of the student's condition, and they can assist in targeting carryover of treatment strategies to treat the paradoxical vocal fold motion disorder (see article by Dr. Sally Gallena and colleagues in this issue for further information). ${ }^{10}$

\section{LINK TO INTERVENTION UNDER IDEA: SERVICE DELIVERY OPTIONS}

There are several service delivery options that are effective under the IDEA guidelines. It is important for clinicians to keep in mind that the typical therapy model that is followed for children with articulation or language-based deficits may be an ineffective model for school-aged children with voice disorders. Therapeutic models should be tailored to fit the academic and therapeutic needs of each student. Clinicians surveyed regarding their caseloads have expressed difficulty finding the best group, time, or service delivery model for a child with a voice disorder. The reason given is because the child with a voice problem may be only 1 of 75 or more of the clinician's caseload. Other questions or concerns that clinicians have raised include: With which therapy group would the student best fit? What treatment activities would fit with the other disorders being treated in the same group? How long do I keep the student in therapy or on the caseload? How do I collect the appropriate data to reflect progress?

Some of the IDEA options for service delivery to address these issues can include:

- Classroom pullout

- Classroom-based service delivery: where the SLP works with the student in the classroom or recreational environment for a designated period of time

- Collaborative: where the SLP works in collaboration with other service delivery team members (i.e., occupational therapist or physical therapist)

- Consultative

- Individual therapy sessions

- Small-group sessions, preferably with other children with voice disorders

The frequency of visits can vary from one time per week every other week to one time per month or a combination. In addition, some unique intervention models can be integrated into service delivery options. Some of these include implementing classroom lessons for the entire class that involve vocal health and prevention strategies; creating science and health projects associated with voice; providing materials that parents can use to teach vocal awareness at home to the entire family; implementing voice treatment programs, with small groups or in peer dyads, or one on one; ${ }^{4}$ and collaborative voice therapy programs with music, drama, or physical education teachers. 


\section{SUMMARY}

This article describes some of the factors that may affect eligibility criteria and seeks to define the role of the SLP and multidisciplinary team working within the framework of IDEA. In addition, triaging scenarios provided practical models of intervention and some unique ways to advocate for students needs with voice disorders, even when the scenario is less than optimal.

Current literature provides more viewpoints regarding the progression of functional and organic voice disorders in school-aged children and the implications of treatment. It is widely advocated that preventative treatment programs should be implemented in school-based voice therapy programs. If left untreated, any voice disorder has the potential to result in more severe structural abnormalities and have a significant impact on academic performance and learning.

\section{REFERENCES}

1. Connor NP, Cohen SB, Theis SM, Thibeault SL, Heatley DG, Bless DM. Attitudes of children with dysphonia. J Voice 2008;22:197-209
2. Ruddy BH, Sapienza CM. Treating voice disorders in the school-based setting: working within the framework of IDEA. Lang Speech Hear Serv Sch 2004;35:327-332

3. U.S. Congress Individuals with Disabilities Education Act amendments of 2004. Washington, D. C.: U.S. Government Printing Office; 2004

4. Andrews M. Voice Treatment for Children and Adolescents. San Diego, CA: Singular Publishing Group, Inc.; 2002

5. Friedberg J. Hoarseness. In: Bluestone C, Sylvan E, eds. Pediatric Otolaryngology. Vol. 2. Philadelphia, PA: W.B. Saunders; 1983:1181-1189

6. Gray SD, Smith ME, Schneider H. Voice disorders in children. Pediatr Clin North Am 1996;43: 1357-1384

7. Glaze L. Treatment of voice hyperfunction in the pre-adolescent. Lang Speech Hear Serv Sch 1996;27:244-250

8. Verdolini K. Critical analysis of common terminology in voice therapy: a position paper. Phonoscope 1999;2:1-8

9. Hirschberg J, Dejonckere PH, Hirano M, Mori K, Schultz-Coulon HJ, Vrticka K. Voice disorders in children. Int J Pediatr Otorhinolaryngol 1995; 32(Suppl):S109-S125

10. Gallena S, Kerins M. Nonspecific chronic cough and paradoxical vocal fold motion disorder in pediatric patients. Semin Speech Lang 2013;34:116-128 